\title{
Erratum: Perturbation treatment of linear beam optics of combined function magnets
} [Phys. Rev. ST Accel. Beams 5, 064001 (2002)]

M. Seidl

(Published 17 July 2002)

DOI: 10.1103/PhysRevSTAB.5.079901

PACS numbers: 29.20.-c, 41.85.Lc, 99.10.+g

In the above article, the following byline footnote was inadvertently omitted: "This work constitutes part of the doctoral thesis of M. Seidl at Universität Mainz, Germany."

I regret the oversight. 